hautnah $2021 \cdot 20: 207-212$

https://doi.org/10.1007/s12326-021-00467-1

Angenommen: 27. September 2021

Online publiziert: 5 . Oktober 2021

(C) Der/die Autor(en) 2021

R. Knobler $\cdot$ T. Valero $\cdot$ U. Just

Universitätsklinik für Dermatologie, Medizinische Universität Wien, Wien, Österreich

\title{
Update zur Extrakorporalen Photopherese
}

benwirkungen. In seltenen Fällen kann es durch Volumenänderungen zu blutdruckassoziierten Symptomen kommen. Bei den modernen Geräten ist auch diese Nebenwirkung nicht mehr aufgetreten.

Kontraindikationen sind eine Unverträglichkeit von 8-MOP, Schwangerschaft und/oder Vorliegen einer ausgeprägten Anämie (Hämatokrit unter $27 \%$ ). Behandlungen von Patienten mit geringem Körpergewicht (z. B. die Behandlung von Kindern) ist durch den Einsatz von modernen Geräten mittlerweile ebenfalls möglich.

Mittlerweile liegen klinische Daten vor, die 3 Jahrzehnte umspannen. Diese zeigen, dass die ECP auch bei langfristiger Anwendung weder eine Toxizität besitzt noch dass die Behandlung mit einem erhöhten Risiko für Infekte oder Malignome vergesellschaftet ist [6].

\section{Phasen der ECP-Behandlung}

Die ECP-Behandlung selbst setzt sich aus den Phasen Leukapherese, Photoaktivierung und Reinfusion zusammen [7]. Vollblut des Patienten wird entweder über einen peripheren oder alternativ - bei schlechter peripherer Venensituation - über einen zentralvenösen Zugang gewonnen. Während der ersten Phase, der Leukapherese, wird das Vollblut zentrifugiert, um die Erythrozyten vom Plasma und von den Leukozyten zu trennen. Die Leukozytenfraktion bildet zusammen mit Plasma und Thrombozyten den sog. „buffy coat“. Diesem wird der Photosensibilisator 8-MOP als sterile Lösung zugesetzt und anschließend zirkuliert der „buffy coat" durch eine dünne Plastikkammer, die Photoaktivierungskammer, die von beiden Seiten mit UVALampen bestrahlt wird.
Am Ende der Bestrahlungszeit werden die behandelten Blutbestandteile dem $\mathrm{Pa}$ tienten rückinfundiert.

\section{Wirkungsweise der Photopherese}

Mittlerweile ist es 35 Jahre her, dass die erste Studie zu ECP abgeschlossen wurde, und 30 Jahre sind seit der Zulassung von ECP durch die US Food and Drug Administration (FDA) vergangen. Trotzdem konnte der Wirkmechanismus, der dem klinischen Erfolg zugrunde liegt, noch nicht vollständig aufgeklärt werden.

Bekannt ist, dass es in lymphoiden Zellen innerhalb von etwa $48 \mathrm{~h}$ durch Kombination von UVA und Psoralen zu DNA-Crosslinking kommt und in weiterer Folge zur Apoptose ebendieser Zellen [8]. Nach Präsentation von Antigenen aus den apoptotischen Leukozyten wird einerseits eine antigenspezifische Immuntoleranz induziert und andererseits kommt es $\mathrm{zu}$ einer tumorspezifischen Immunreaktion.

Neueren Erkenntnissen zufolge beruhen die immunmodulatorischen Effekte auf einer Differenzierung von Monozyten in dendritische antigenpräsentierende Zellen (DCs), einer Änderung der Zytokinprofile mit Switch zwischen Th1 und Th2 sowie auf der Induktion von regulatorischen T-Zell-Subpopulationen.

Monozyten des peripheren Bluts, die mittels ECP behandelt werden, gehen nicht in Apoptose sondern exprimieren Marker an der Oberfläche, die charakteristisch für unreife dendritische Zellen sind. Edelson et al. vermuten, dass diese Differenzierung in unreife dendritische Zellen mit nachfolgender Phagozytose von ebenfalls rückinfundierten apoptotischen Lymphozyten durch den Kontakt tienten im Allgemeinen gut vertragen, und es gibt fast keine unerwünschten $\mathrm{Ne}$ - 

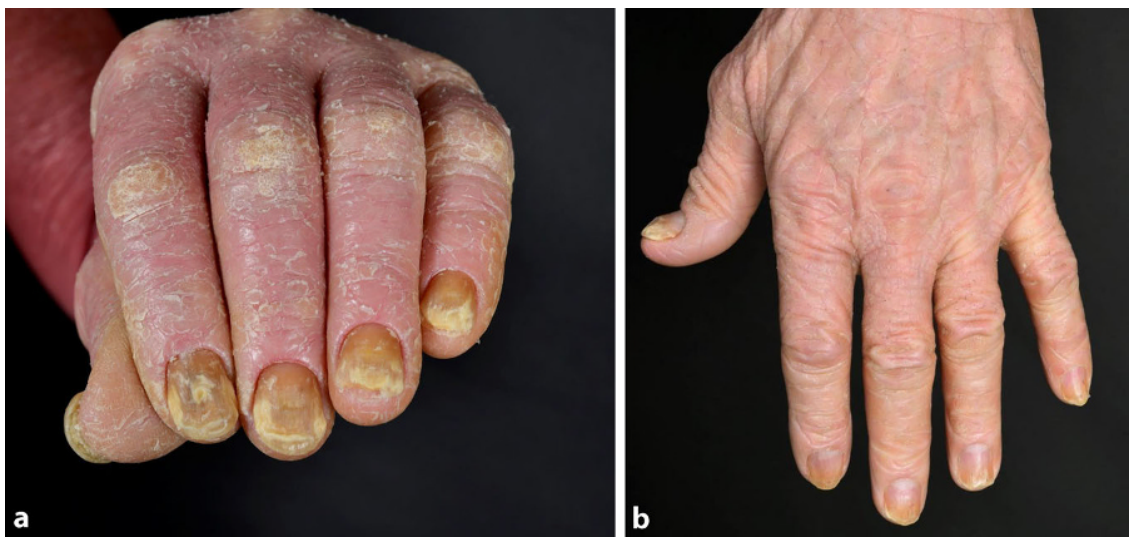

Abb. $1 \Delta$ a Hände und Nägel eines Patienten mit CTCL vor ECP. b Nach 16 Monaten ECP-Behandlung

zu den Kunststoffmaterialien des Photopheresesystems bedingt ist und bezeichnen diesen Prozess auch als „Transimmunisierung“ [9].

Anschließend kommt es zur Reifung in differenzierenden dendritischen Zellen und Präsentation von antigenen Peptiden [10-12]. Die Phagozytose der malignen Zellpopulation bei CTCL durch dendritische Zellen scheint durch Aktivierung von antitumorspezifischen T-Zellen zytotoxische Reaktionen gegen T-Zell-Klone auszulösen [13].

Auch kommt es nach der Reinfusion von 8-MOP- und UVA-behandelten Zellen $\mathrm{zu}$ einem Anstieg von Tumornekrosefaktor(TNF)-alpha und IL-6 im peripheren Blut [14], was wiederum zur Induktion von CD36+-Monozyten führt.

Eine wichtige Rolle spielen immunologische Reaktionen, die nach wiederholten ECP-Behandlungen auftreten. Im Fall von CTCL kommt es im Rahmen der Erkrankung zu einem Ungleichgewicht der Th1/Th2-Immunantwort, wodurch es zu einer vermehrten Freisetzung von Interleukinen (IL-4 und IL-5), einer reduzierten Aktivität natürlicher Killerzellen und einer reduzierten Zytotoxizität CD8-positiver T-Zellen kommt $[15,16]$.

Nach einer einjährigen ECP-Behandlung konnte nachgewiesen werden, dass das Ungleichgewicht der Th1/Th2-Reaktion normalisiert werden konnte, entzündungshemmende Zytokine durch ECP induziert werden, während proinflammatorische Zytokine reduziert werden [17]. Diese Mechanismen können die Wirkung der ECP bei CTCL erklären, der Mechanismus bei GvHD und anderen Autoimmunerkrankungen, bei denen ein immunsuppressiver Effekt eine Rolle spielt, müsste einem anderen Weg folgen.

\section{》) Bei Patienten mit GvHD verschiebt die ECP das Zytokinprofil in Richtung einer Th2-Immunantwort}

In der Tat, bei Patienten mit GvHD verschiebt die ECP das Zytokinprofil in Richtung einer Th2-Immunantwort. Vergleicht man die Zytokinprofile von Patienten vor und nach ECP, kann man einen Anstieg von IL-4, IL-10 und Transforming-Growth-Factor(TGF)-Beta und eine Abnahme von IL-12, IL-1, Interferon- $\alpha$ und TNF- $\alpha$ beobachten, was zur Apoptose von mononukleären Zellen führt $[18,19]$.

Überdies werden durch die ECP regulatorische T-Zellen (Tregs) induziert, welche die Fähigkeit haben, andere Lymphozyten zu supprimieren, und damit eine wichtige Rolle bei der Regulierung von Immunreaktionen spielen [20]. Tregs sind durch die Expression von CD4, CD25, CTLA-4 und den Transkriptionsfaktor FOXP3 charakterisiert.

Es ist bekannt, dass Tregs bei Patienten mit Sézary-Syndrom vermindert und in ihrer Funktion gestört sind [21, 22]. Auch im Rahmen einer akuten GvHD bei Patienten mit allogener Knochenmarktransplantation kommt es zu einer erniedrigten Zahl an Tregs [21].
Mehrfach konnte bisher gezeigt werden, dass eine längerdauernde ECPBehandlung sowohl zu einem Anstieg der Tregs als auch zu einer gesteigerten suppressiven Wirkung der Tregs von CTCL- und GvHD-Patienten führt [21, 22]. Es besteht die Annahme, dass Tregs einerseits die CD4-positiven Tumorzellen beim Sézary-Syndrom und andererseits die bei der GvHD autoimmun reagierenden $\mathrm{T}$-Zellen supprimieren können.

Zusammenfassend lässt sich sagen, dass bisherige Erkenntnisse nahelegen, dass es durch Rückinfusion von apoptotischen Zellen zu einem immunmodulatorischen Effekt kommt, der auf der Differenzierung dendritischer Zellen, einer Änderung des Zytokinprofils und Stimulation von regulatorischen T-Zellen basiert. Verschiedene Mechanismen tragen zu den positiven Wirkungen von ECP bei verschiedenen Indikationen bei. Jedoch ist die genaue Rolle, die regulatorische T-Zellen spielen, noch nicht endgültig geklärt.

\section{Kutane T-Zell-Lymphome}

Kutane T-Zell-Lymphome („cutaneous T-cell lymphomas“, CTCL) bezeichnen eine heterogene Gruppe von lymphoproliferativen Erkrankungen der Haut. Die häufigste Form ist die sog. Mycosis fungoides (MF). Eine seltene Variante (etwa $5 \%)$ ist das Sézary-Syndrom mit Erythrodermie, Leukämie und Lymphknotenbefall. Das Sézary-Syndrom hat eine schlechte Prognose mit einer mittleren Überlebensrate von etwa 3 Jahren.

Als erste Publikation wurde im Jahr 1987 im New England Journal of Medicine (NEJM) von Edelson und seinen Mitarbeitern [1] erstmals über eine vielversprechende Therapiestrategie zur Behandlung therapierefraktärer kutaner T-Zell-Lymphome (CTCL) berichtet. Die therapeutischen Erfolge dieser ersten Studie konnten in den folgenden Jahren und bis zum heutigen Tag wiederholt bestätigt werden (• Abb. 1). Patienten mit der erythrodermatischen Variante dieser Erkrankungsgruppe mit intaktem Immunsystem, mit einem noch relativ niedrigen CD4:CD8-Verhältnis scheinen am besten auf die ECP als 
Mono- oder als Kombinationstherapie anzusprechen [23, 24]. Rezente klinische Untersuchungen deuten darauf hin, dass Kombinationstherapien von ECPBehandlungen mit Immunochemotherapien, Interferontherapien wie auch fast allen anderen Therapien die Ansprechrate weiter erhöhen können.

In einer Vielzahl von Studien konnten Ansprechraten von ECP in der Behandlung von CTCL von $70 \%$ gezeigt werden. In Zukunft sollen auch prospektive Studien die Wirkung und Effektivität der ECP bei frühen Formen (Plaque-Stadium) der MF untersuchen [25].

\section{》) Im Frühstadium des Sézary-Syndroms ist die Therapieerfolgsquote deutlich erhöht}

Es konnte gezeigt werden, dass im Frühstadium des Sézary-Syndroms die Therapieerfolgsquote deutlich erhöht ist. In der Identifizierung von möglichen Therapieversagern ist einerseits die Krankheitsdauer, andererseits die Tumorausdehnung in Betracht zu ziehen. Beste Ansprechraten zeigen CTCL-Patienten mit einer Krankheitsdauer von unter 2 Jahren und ohne Lymphknotenbefall oder Befall innerer Organe. Ebenso kann man bei einer Leukozytose unter 20.000 Zellen pro $\mathrm{mm}^{3}$ mit einem prozentualen Anteil von weniger als 10-20\% SézaryZellen mit einem erhöhten Therapieerfolg mittels ECP rechnen. Eine normale Natural-Killer-Zell-Aktivität und eine weitgehend im Normbereich liegende Anzahl von zytotoxischen T-Zellen, wobei CD8-positive T-Suppressorzellen anteilsmäßig mehr als $15 \%$ ausmachen sollten, lassen vor Therapiebeginn mit ECP auf einen Therapieerfolg schließen [26-28]. Im Laufe der letzten Jahre konnte auch belegt werden, dass Patienten, die in Folge früherer Therapiestrategien multiple chemotherapeutische Behandlungen erhalten hatten, häufig nicht optimal auf die ECP ansprechen.

hautnah 2021 -20:207-212 https://doi.org/10.1007/s12326-021-00467-1

(c) Der/die Autor(en) 2021

\section{R. Knobler $\cdot$ T. Valero $\cdot$ U. Just}

\section{Update zur Extrakorporalen Photopherese}

\section{Zusammenfassung}

Vor über 30 Jahren wurde ein von der PUVA abgeleitetes Therapiekonzept zur Behandlung von therapierefraktären kutanen T-Zell-Lymphomen (CTCL), insbesondere dem Sézary-Syndrom, entwickelt. R. Edelson hat Leukapherese mit Photochemotherapie verbunden, wodurch Zellen des peripheren Blutes extrakorporal nach Photosensibilisierung mittels Psoralen mit UVA-Licht bestrahlt und anschließend rückinfundiert werden. Im Jahr 1987 wurde dieses neue Behandlungskonzept mit dem Namen extrakorporale Photopherese (ECP) erstmals publiziert. Nach den ersten positiven Erfahrungen mit CTCL wurde diese Behandlung bei weiteren Indikationen erfolgreich angewendet, darunter Erkrankungen wie akute und chronische Graft-versus-HostReaktion (GvHD), Sklerodermie sowie atopische Dermatitis (AD). Eine zunehmende Anwendung hat die ECP in den letzten Jahren in der Behandlung von akuten und chronischen Abstoßungsreaktionen nach Organtransplantation, insbesondere Herzund Lungentransplantation, erhalten. Im Jahr 2021 gibt es über 200 Zentren weltweit, welche die ECP erfolgreich einsetzen.

\section{Schlüsselwörter}

Leukapherese · Photochemotherapie · Kutane T-Zell-Lymphome (CTCL) · Mycosis fungoides . Sézary-Syndrom · Sklerodermie · Graft-versusHost-Erkrankung (GvHD)

\section{Update on Extracorporeal Photopheresis}

\section{Abstract}

More than 30 years ago, ECP (extracorporeal photochemotherapy) was developed for the treatment of refractory cutaneous T-cell lymphomas (CTCL), in particular Sézary syndrome. R. Edelson combined leukapheresis with photochemotherapy, where cells of the peripheral blood are irradiated extracorporeally with UVA light after photosensitization using psoralen and then reinfused. This new treatment concept was first published as extracorporeal photopheresis (ECP) in 1987. After initial positive experience with $C T C L$, this treatment was successfully applied to other indications, including acute and chronic graft-versus- host disease (GvHD), scleroderma, and atopic dermatitis (AD). ECP has been increasingly used in recent years for the treatment of acute and chronic rejection after organ transplantation, especially heart and lung transplantation. As of 2021, there are over 200 centers worldwide that successfully use ECP.

\section{Keywords}

Leukapheresis - Photochemotherapy . Cutaneous T-cell lymphoma - Mycosis fungoides · Sézary syndrome - Scleroderma . Graft-versus-Host-Disease (GvHD)

\section{Akute und chronische Graft- versus-Host-Erkrankung}

Obwohl die allogene Stammzelltransplantation eine potenziell kurative Behandlung darstellt, ist die GvHD immer noch der limitierende Faktor für den Krankheitsverlauf dieser Patienten [29]. Nach dem ersten Bericht im Jahr 1994 [30] über die erfolgreiche Anwendung der ECP bei der GvHD-Erkrankung wurde die Wirksamkeit der ECP-Behandlung bei GvHD in multiplen Studien bestätigt [31-33].
Im Rahmen von Metaanalysen von 31 durchgeführten Studien wurde die Photopherese zur Behandlung der akuten wie chronischen GvHD nach Knochenmarktransplantation (KMT) weiter untersucht. Vorrangig wurden GvHDPatienten behandelt, deren Symptome durch eine konventionelle Therapiestrategie nicht kontrolliert werden konnten [34].

Bei der Anwendung der ECP bei der akuten GvHD konnte gezeigt werden, dass es zu einer signifikanten Linderung der kutanen Manifestationen um etwa $83 \%$ kommt. Bei zusätzlichem Befall 
innerer Organe (Lunge, Leber) und der Mundschleimhaut konnte durch eine ECP-Behandlung auch eine Besserung der Symptome dokumentiert werden. Bei Auftreten einer chronischen GvHD kam es nach einer Behandlungsdauer von 3-40 Monaten zu einer Regression der Hauterscheinungen bei $76 \%$ der Patienten. Erscheinungsfreiheit an der Haut wurde in $38 \%$ dokumentiert. Die Überlebensrate der Patienten lag bei $79 \%$.

In weiteren Studien konnte gezeigt werden, dass es sich bei der ECP um eine zusätzliche effektive adjuvante Therapie für die steroidrefraktäre GvHD nach KMT mit Haut- und Lebermanifestationen handelt. Weniger wirksam war diese als Begleittherapie bei sehr fortgeschrittenen Stadien der GvHD (Stadium IV) sowie bei Vorliegen ausgeprägter gastrointestinaler Beteiligung [35, 36]. Diese zahlreichen Berichte zeigen, dass es sich bei der ECP um eine effektive, steroidsparende und praktisch nebenwirkungsfreie Therapie zur Behandlung sowohl der akuten als auch der chronischen GvHD handelt.

\section{Systemische Sklerose}

Nach wie vor werden bei der systemischen Sklerose autoimmunologische Prozesse als pathogenetische Mechanismen in Betracht gezogen. Diese Überlegungen werden auch durch den Nachweis von infiltrierenden T-Zellen und autoreaktiven Antikörpern in den betroffenen Organen gestützt. Ein einheitliches pathophysiologisches Konzept der systemischen Sklerose gibt es bis dato nicht.

Auch ist die Therapie der systemischen Sklerose bis heute relativ unbefriedigend und richtet sich zumeist nach der Schwere des Krankheitsbildes bzw. den befallenen Organsystemen. Bisherige Therapieansätze konnten weder anhaltende Remissionen erzielen noch konnte eine Verlängerung der Überlebenszeit erreicht werden. Im Rahmen der ECP kommt es zur einer signifikanten Erhöhung von Tumornekrosefaktor (TNF), und im Rahmen von Studien konnte gezeigt werden, dass bei der systemischen
Sklerose erhöhte TNF-Spiegel zu einer verminderten Kollagensynthese führen.

In einer kontrollierten prospektiven klinischen Studie konnte relativ früh nach der Entwicklung der ECP gezeigt werden, dass die Photopherese eine gut verträgliche Therapieoption mit einer positiven Beeinflussung des Krankheitsverlaufs darstellt [37]. In dieser Studie wurden Patienten mindestens über 6 Monate randomisiert behandelt, entweder mit D-Penicillamin oder ECP. Nachfolgend zeigten Skin-Score-Messungen eine signifikante Verbesserung der mit ECP behandelten Patientengruppe. Diese Verbesserungen konnten auch mittels Hautbiopsien histologisch verifiziert werden.

Im Verlauf einer langfristigen Photopherese-Therapie zur Behandlung der systemischen Sklerose konnte nicht nur nachgewiesen werden, dass es zu einer Stabilisierung oder sogar Verbesserung des Krankheitsverlaufs bezüglich der Hautveränderungen kommt [4], sondern auch zu einer signifikanten Verlängerung der Überlebenszeit [38]. Im Gegensatz zu den Hautmanifestationen konnten viszerale Manifestationen selbst durch lang andauernde Behandlungen kaum beeinflusst werden. Eine positive Beeinflussung des Krankheitsverlaufs war umso deutlicher, je früher mit der ECP-Therapie begonnen wurde. Im Rahmen einer prospektiven Studie [39] konnte nachgewiesen werden, dass das Vorhandensein einer klonalen T-ZellPopulation im Rahmen der systemischen Sklerose mit einer besseren Ansprechrate auf ECP vergesellschaftet ist.

\section{Allograft-Abstoßung}

Die Überlebenswahrscheinlichkeit nach Allografttransplantationen beruht auf der Kontrolle der Abstoßungsreaktion. Diese richtet sich vornehmlich gegen Spendermoleküle des Major-Histocompatibility-Komplexes (MHC). Die Unterdrückung der Abstoßungskaskade ist essenziell für Patienten nach Organtransplantation. Nachdem sich die Photopherese in der Behandlung von akuter sowie chronischer GvHD als erfolgreich erwiesen hatte, wurden auch klinische Studien durchgeführt, in denen Patienten nach Organtransplantationen einer ECP-Be- handlung unterzogen wurden [40]. Bei Patienten mit einer Abstoßungsreaktion nach Herztransplantation (HTX) konnte gezeigt werden, dass durch ECPBehandlungen sowohl histologisch als auch klinisch eine deutliche Besserung der kardialen Situation erreicht werden kann.

In einer weiteren Vergleichsstudie von ECP und Kortikosteroiden bei der Kontrolle der Organabstoßungsreaktion nach HTX konnte nachgewiesen werden, dass die Wirksamkeit der ECP mit der von Kortikosteroiden vergleichbar ist. Das Nebenwirkungsprofil der ECP im Vergleich mit dem der Kortikosteroide ist allerdings eindeutig geringer [41].

Im Management der Abstoßungsreaktionen nach Herztransplantationen ist die Photopherese mittlerweile als adjuvante Therapie anerkannt. Sowohl bei der Behandlung von akuten Abstoßungen als Alternative zu einer Kortikosteroidstoßtherapie als auch in der chronischen Abstoßungsreaktion wird die ECP mit zunehmendem Erfolg eingesetzt. In mehreren klinischen Studien nach Herztransplantationen konnte einerseits festgestellt werden, dass es zu signifikant weniger Abstoßungsereignissen kommt $[42,43]$ und man andererseits eine Dosisreduktion der immunsuppressiven Therapie in Kombination mit einer Photopherese-Behandlung erreichen kann. Obwohl es bei Patienten mit oder ohne ECP-Behandlung keine Unterschiede in der Häufigkeit von Infektionen gab, konnte doch Zytomegalievirus-DNA deutlich weniger oft in der ECP-Gruppe nachgewiesen werden.

Somit konnte bewiesen werden, dass die adjuvante ECP nach einer Herztransplantation das Risiko der Organabstoßung reduziert bzw. eine bereits bestehende Abstoßung verbessern kann, ohne dass es zu einer erhöhten Inzidenz von Zytomegalievirus-Infektionen führt.

Das Auftreten eines Bronchiolitis-obliterans-Syndroms (BOS) bei Patienten nach Lungentransplantation ist eine nicht seltene und auch schwer therapierbare Komplikation. Eine signifikante Verbesserung der Symptomatik und des Gesamtüberlebens durch den Einsatz der ECP in der Behandlung des BOS konnte bereits in einigen klinischen Studien ge- 
zeigt werden [44]. Neueste Studien weisen darauf hin, dass der frühzeitige Einsatz der ECP, kurzfristig nach der Transplantation selbst, dem Auftreten einer BOS prophylaktisch entgegenwirkt.

\section{Fazit}

- Die ECP hat sich, seit der Entwicklung durch Dermatologen vor über 30 Jahren, zu einer etablierten fachübergreifenden Therapie entwickelt.

- Aufgrund des hervorragenden Sicherheitsprofils erfährt die ECP zunehmende Akzeptanz als effektive immunmodulatorische Therapie.

- Vielversprechende therapeutische Erfolge konnten bei einem sich ständig erweiternden Indikationsspektrums erzielt werden $[45,46]$.

- Prospektive randomisierte Studien mit größeren Kohorten sind allerdings erforderlich, einerseits um die Komplexität der zugrundeliegenden immunmodulatorischen Mechanismen besser zu verstehen und andererseits zur Identifizierung von Biomarkern, die möglicherweise das Ansprechen auf die Behandlung anzeigen können.

\section{Korrespondenzadresse}

\section{Prof. Dr. R. Knobler}

Universitätsklinik für Dermatologie, Medizinische Universität Wien Währinger Gürtel 18-20, 1090 Wien, Österreich robert.knobler@meduniwien.ac.at

Funding. Open access funding provided by Medical University of Vienna.

\section{Einhaltung ethischer Richtlinien}

Interessenkonflikt. R. Knobler und U. Just haben unabhängig von dieser Arbeit persönliche speaker Honorare von Mallinckrodt/Therakos erhalten. T. Valero gibt an, dass kein Interessenkonflikt besteht.

Für diesen Beitrag wurden von den Autoren keine Studien an Menschen oder Tieren durchgeführt. Für die aufgeführten Studien gelten die jeweils dort angegebenen ethischen Richtlinien.

Open Access. Dieser Artikel wird unter der Creative Commons Namensnennung 4.0 International Lizenz veröffentlicht, welche die Nutzung, Vervielfältigung, Bearbeitung, Verbreitung und Wiedergabe in jeglichem Medium und Format erlaubt, sofern Sie den/die ursprünglichen Autor(en) und die Quelle ordnungsgemäß nennen, einen Link zur Creative Commons Lizenz beifügen und angeben, ob Änderungen vorgenommen wurden.

Die in diesem Artikel enthaltenen Bilder und sonstiges Drittmaterial unterliegen ebenfalls der genannten Creative Commons Lizenz, sofern sich aus der Abbildungslegende nichts anderes ergibt. Sofern das betreffende Material nicht unter der genannten Creative Commons Lizenz steht und die betreffende Handlung nicht nach gesetzlichen Vorschriften erlaubt ist, ist für die oben aufgeführten Weiterverwendungen des Materials die Einwilligung des jeweiligen Rechteinhabers einzuholen.

Weitere Details zur Lizenz entnehmen Sie bitte der Lizenzinformation auf http://creativecommons.org/ licenses/by/4.0/deed.de.

\section{Literatur}

1. Edelson R, Berger C, Gasparro F, Jegasothy $B$, Heald $P$, Wintroub $B$, Vonderheid $E$, Knobler $R$ Wolff K, Plewig G (1987) Treatment of cutaneous T-cell lymphoma by extracorporeal photochemotherapy. Preliminary results. N Engl J Med 316:297-303

2. Bladon J, Taylor PC (1999) Extracorporeal photopheresis induces apoptosis in the lymphocytes of cutaneous T-cell lymphoma and graft-versus-host disease patients. Br J Haematol 107:707-711

3. Greinix HT, Volc-Platzer B, Knobler RM (2000) Extracorporeal photochemotherapy in the treatment of severe graft-versus-host disease. Leuk Lymphoma 36:425-434

4. Knobler RM, French LE, Kim Y, Bisaccia E, Graninger W, Nahavandi $\mathrm{H}$, Strobl FJ, Keystone $\mathrm{E}_{\text {, }}$ Mehlmauer M, Rook AH, Braverman I, Systemic Sclerosis Study Group (2006) A randomized, double-blind, placebo-controlled trial of photopheresis in systemic sclerosis. J Am Acad Dermatol 54:793-799

5. Prinz B, Nachbar F, Plewig G (1994) Treatment of severe atopic dermatitis with extracorporeal photopheresis. Arch Dermatol Res 287:48-52

6. Topuzoglu S, Knobler R, Movadat O, Petkov V, Foedinger $\mathrm{D}$, Just $\mathrm{U}$, Pehamberger $\mathrm{H}$, Jantschitsch $\mathrm{C}$ (2015) Incidence of lung cancer in patients with systemic sclerosis treated with extracorporeal photopheresis. Photodermatol Photoimmunol Photomed 31:175-183

7. Edelson RL (1989) Photopheresis: a new therapeutic concept. Yale JBiol Med 62:565-577

8. YooEK, RookAH, Elenitsas R, GasparroFP, Vowels BR (1996) Apoptosis induction by ultraviolet light $A$ and photochemotherapy in cutaneous T-cell lymphoma: relevance to mechanism of therapeutic action. J Invest Dermatol 107:235-242

9. Berger $\mathrm{CL}$, Hanlon $\mathrm{D}$, Kanada D, Girardi $\mathrm{M}$, Edelson RL (2002) Transimmunization, a novel approach for tumor immunotherapy. Transfus Apher Sci 26:205-216

10. Edelson R, Wu Y, Schneiderman J (2018) American council on ECP (ACE): why now? J Clin Apheresis 33:464-468

11. Berger $\mathrm{CL}$, $\mathrm{Xu} A \mathrm{~L}$, Hanlon $\mathrm{D}$, Lee $\mathrm{C}$, Schechner J, Glusac E, Christensen I, Snyder E, Holloway V, Tigelaar R, Edelson RL (2001) Induction of human tumor-loaded dendritic cells. Int J Cancer 91:438-447

\section{Dr. Volker Hahn \\ Die souveräne Expertin}

Heidelberg: Springer Verlag 2020,

213 S., 196 Abb., (ISBN: 978-3-662-

61722-9), 22,99 EUR

Tipps für die Wissenschaftskommunikation und praktische Hinweise für verständliches Erklären

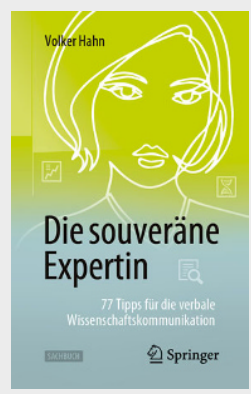

Dieses Buch richtet sich an alle, die ihre wissenschaftlichen Themen anschaulich und verständlich kommunizieren möchten. Der Autor Volker Hahn wählte für sein

Buch und den dazugehörigen Titel zwar die weibliche Form, spricht aber alle Menschen an, die forschen oder geforscht haben.

In der Wissenschaft nicht zu kommunizieren ist nicht möglich...

...und gut zu kommunizieren ist nicht einfach. Fachexpertise allein reicht nicht aus, um verständlich zu erklären, souverän vorzutragen und erfolgreich in den Medien aufzutreten. Dabei bergen wissenschaftliche Themen ganz spezifische Herausforderungen. Verbale Wissenschaftskommunikation ist eine Querschnittsaufgabe aus den Bereichen Strategie, Erklären und Erzählen, Rhetorik und Körpersprache. Für alle diese Bereiche vermittelt das Buch praktische Tipps mit zahlreichen Beispielen aus der Praxis. Es hilft dabei, zur souveränen Expertin oder zum souveränen Experten zu werden - in Interviews, nicht nur in den Medien, in Vorträgen, Gesprächen und Diskussionsrunden. 77 kurze und voneinander unabhängige Tipps machen das Buch ideal für die Lektüre zwischendurch.

\section{Der Autor:}

Dr. Volker Hahn hat in verschiedenen Rollen auf dem Feld der Wissenschaftskommunikation gearbeitet: als Biogeochemiker in der Wissenschaft, als Journalist u.a. bei 3sat und SWR, als Medientrainer und als Leiter einer Forschungspressestelle. 
12. Girardi $M$, Berger $C L$, Wilson LD, Christensen IR, Thompson KR, Glusac EJ, Edelson RL (2006) Transimmunization for cutaneous T cell lymphoma: A phase Istudy. Leuk Lymphoma 47:1495-1503

13. Ventura $A$, Vassall $A$, Robinson E, Filler $R$, HanIon D, Meeth K, Ezaldein H, Girardi M, Sobolev O, Bosenberg MW, Edelson RL (2018) Extracorporeal photochemotherapy drives monocyte-to-dendritic cell maturation to induce anticancer immunity. Cancer Res 78:4045-4058

14. Vowels BR, Cassin M, Boufal MH, Walsh LJ, RookAH (1992) Extracorporeal photochemotherapy induces the production of tumor necrosis factor-alpha by monocytes: implications for the treatment of cutaneous T-cell lymphoma and systemic sclerosis. J Invest Dermatol 98:686-692

15. Bladon J, Taylor PC (2006) Extracorporeal photopheresis: a focus on apoptosis and cytokines. JDermatol Sci 43:85-94

16. Voss CY, Fry TJ, Coppes MJ, Blajchman MA (2010) Extending the horizon for cell-based immunotherapy by understanding the mechanisms of action of photopheresis. Transfus Med Rev 24:22-32

17. Di Renzo M, Rubegni P, De Aloe G, Paulesu L, Pasqui AL, Andreassi L, Auteri A, Fimiani M (1997) Extracorporeal photochemotherapy restores Th1/Th2 imbalance in patients with early stage cutaneous T-cell lymphoma. Immunology 92:99-103

18. Berger C, Hoffmann K, Vasquez JG, Mane S, Lewis J, Filler R, Lin A, Zhao H, Durazzo T, Baird A, Lin W, Foss F, Christensen I, Girardi M, Tigelaar R, Edelson R (2010) Rapid generation of maturationally synchronized human dendritic cells: contribution to the clinical efficacy of extracorporeal photochemotherapy. Blood 116:4838-4847

19. Rao V, Saunes M, Jørstad S, Moen T (2009) Cutaneous $T$ cell lymphoma and graft-versushost disease: a comparison of in vivo effects of extracorporeal photochemotherapy on Foxp3+ regulatory T cells. Clin Immunol 133:303-313

20. Maeda A Schwarz A, Kernebeck K, Gross N Aragane Y, Peritt D, Schwarz T (2005) Intravenous infusion of syngeneic apoptotic cells by photopheresis induces antigen-specific regulatory $T$ cells. JImmunol 174:5968-5976

21. Quaglino $P$, Comessatti A, Ponti R, Peroni $A$ Mola F, Fierro MT, Savoia $P$, Novelli M, Bernengo MG (2009) Reciprocal modulation of circulating CD4+CD25+bright T cells induced by extracorporeal photochemotherapy in cutaneous T-cell lymphoma and chronic graft-versus-hostdisease patients. Int J Immunopathol Pharmacol 22:353-362

22. Di Biaso I, Di Maio L, Bugarin C, Gaipa G, Dander E, Balduzzi A, Parma M, D'Amico G, Perseghin P, Biondi A, Biagi E (2009) Regulatory T cells and extracorporeal photochemotherapy: correlation with clinical response and decreased frequency of proinflammatory $\mathrm{T}$ cells. Transplantation 87:1422-1425

23. ScarisbrickJJ, TaylorP, HoltickU, MakarY,DouglasK, Berlin G, Juvonen E, Marshall S, Photopheresis Expert Group (2008) U.K. consensus statement on the use of extracorporeal photopheresis for treatment of cutaneous T-cell lymphoma and chronic graft-versus-host disease. Br J Dermatol 158:659-678

24. Stadler R, Assaf C, Klemke C-D, Nashan $D$ Weichenthal M, Dummer R, Sterry W (2008) Short German guidelines: cutaneous lymphomas. JDtsch Dermatol Ges 6(Suppl 1):S25-S31
25. Lewis DJ, Duvic M (2017) Extracorporeal photopheresis for the treatment of early-stage mycosis fungoides. Dermatol Ther 30(3)

26. Zic JA (2015) Extracorporeal photopheresis in the treatment of mycosis fungoides and Sézary syndrome. Dermatol Clin 33:765-776

27. Photiou L, van der Weyden C, McCormack C, Prince HM (2018) Systemic treatment options for advanced-stage mycosis fungoides and Sézary syndrome. Curr Oncol Rep 20:32

28. Bisaccia E, Gonzalez J, Palangio M, Schwartz J, Klainer AS (2000) Extracorporeal photochemotherapy alone or with adjuvant therapy in the treatment of cutaneous T-cell lymphoma: a 9-year retrospective study at a single institution. J Am Acad Dermatol 43:263-271

29. Socié G, Stone JV, Wingard JR, WeisdorfD, HensleeDowney PJ, Bredeson C, Cahn JY, Passweg JR, Rowlings PA, Schouten HC, Kolb HJ, Klein JP (1999) Long-term survival and late deaths after allogeneic bone marrow transplantation. Late Effects Working Committee of the International Bone Marrow Transplant Registry. N Engl J Med 341:14-21

30. Owsianowski M, Gollnick H, Siegert W, Schwerdtfeger R, Orfanos CE (1994) Successful treatment of chronic graft-versus-host disease with extracorporeal photopheresis. Bone Marrow Transplant 14:845-848

31. Child FJ, Ratnavel R, Watkins $P$, Samson $D$ Apperley J, Ball J, Taylor P, Russell-Jones R (1999) Extracorporeal photopheresis (ECP) in the treatment of chronic graft-versus-host disease (GVHD). Bone Marrow Transplant 23:881-887

32. Flowers MED, Apperley JF, van Besien K, Elmaagacli A, Grigg A, Reddy V, Bacigalupo A, Kolb H-J, Bouzas L, Michallet M, Prince HM, Knobler R, Parenti D, Gallo J, Greinix HT (2008) A multicenter prospective phase 2 randomized study of extracorporeal photopheresis for treatment of chronic graft-versus-host disease. Blood 112:2667-2674

33. Greinix HT, Socié $G$, Bacigalupo A, Holler $E$, Edinger MG, Apperley JF, Schwarz T, Ullrich SE, Albert ML, Knobler RM, Peritt D, Ferrara JLM (2006) Assessing the potential role of photopheresis in hematopoietic stem cell transplant. Bone Marrow Transplant 38:265-273

34. Greinix HT, Worel N, Knobler R (2010) Role of extracorporeal photopheresis (ECP) in treatment of steroid-refractory acute graft-versus-host disease. Biol Blood Marrow Transplant 16:1747-1748 (author reply 1749.)

35. Greinix HT, Worel N, Just U, Knobler R (2014) Extracorporeal photopheresis in acute and chronic graft-versus-host disease. Transfus Apher Sci 50:349-357

36. Wolff $D$, Schleuning $M$, von Harsdorf $S$, Bacher $U$, Gerbitz A, Stadler M, Ayuk F, Kiani A, Schwerdtfeger R, Vogelsang GB, Kobbe G, Gramatzki M, Lawitschka A, Mohty M, Pavletic SZ, Greinix H, Holler E (2011) Consensus conference on clinical practice in chronic GVHD: second-line treatment of chronic graft-versus-host disease. Biol Blood Marrow Transplant 17:1-17

37. Rook AH, Freundlich $B$, Jegasothy $B V$, Perez $M I^{\prime}$ Barr WG, Jimenez SA, Rietschel RL, Wintroub B, Kahaleh MB, Varga J (1992) Treatment of systemic sclerosis with extracorporeal photochemotherapy. Results of a multicenter trial. Arch Dermatol 128:337-346

38. Gambichler T, Özsoy O, Bui D, Scheel CH, Susok L (2021) Preliminary results on long-term follow-up of systemic sclerosis patients under extracorporeal photopheresis. J Dermatolog Treat. https://doi. org/10.1080/09546634.2021.1925078

39. French LE, Lessin SR, Addya K, Denardo $B$ Margolis DJ, Leonard DG, Rook AH (2001) Identification of clonal $\mathrm{T}$ cells in the blood of patients with systemic sclerosis: positive correlation with response to photopheresis. Arch Dermatol 137:1309-1313

40. Barr ML, Meiser BM, Eisen HJ, Roberts RF, Livi U, Dall'Amico R, Dorent R, Rogers JG, Radovančević B, Taylor DO et al (1998) Photopheresis for the prevention of rejection in cardiac transplantation. NEngl JMed 339:1744-1751

41. Marques MB, Schwartz J (2011) Update on extracorporeal photopheresis in heart and lung transplantation.J Clin Apheresis 26:146-151

42. Barr ML, Baker CJ, Schenkel FA, McLaughlin SN Stouch BC, Starnes VA, Rose EA (2000) Prophylactic photopheresis and chronic rejection: effects on graft intimal hyperplasia in cardiac transplantation. Clin Transplant 14:162-166

43. Dall'Amico R, Murer L (2002) Extracorporeal photochemotherapy: a new therapeutic approach fo allograft rejection. Transfus Apher Sci 26:197-204

44. Jaksch P, Scheed A, Keplinger M, Ernst M-B, Dani T, Just U, Nahavandi $H$, Klepetko W, Knobler R (2012) A prospective interventional study on the use of extracorporeal photopheresis in patients with bronchiolitis obliterans syndrome after lung transplantation. J Heart Lung Transplant 31:950-957

45. Knobler $R$, Arenberger $P$, Arun $A$, Assaf $C$, Bagot $M$, Berlin G, Bohbot A, Calzavara-Pinton P, Child F, Cho A, French LE, Gennery AR, Gniadecki R, Gollnick HPM, Guenova E, Jaksch P, Jantschitsch C, Klemke C, Ludvigsson J, Papadavid E, Scarisbrick J, Schwarz T, Stadler R, Wolf P, Zic J, Zouboulis C, Zuckermann A, Greinix H (2020) European dermatology forum - updated guidelines on the use of extracorporeal photopheresis 2020 - part 1.J Eur Acad Dermatol Venereol 34:2693-2716

46. Knobler $R$, Arenberger $P$, Arun $A$, Assaf $C$, Bagot $M$, Berlin G, Bohbot A, Calzavara-Pinton P, Child F, Cho A, French LE, Gennery AR, Gniadecki R, Gollnick HPM, Guenova E, Jaksch P, Jantschitsch C, Klemke C, Ludvigsson J, Papadavid E, Scarisbrick J, Schwarz T, Stadler R, Wolf P, Zic J, Zouboulis C, Zuckermann A, Greinix H (2021) European dermatology forum: Updated guidelines on the use of extracorporeal photopheresis 2020_Part 2. JEur Acad Dermatol Venereol 35:27-49

Hinweis des Verlags. Der Verlag bleibt in Hinblick auf geografische Zuordnungen und Gebietsbezeichnungen in veröffentlichten Karten und Institutsadressen neutral. 